\title{
Etude de la réactivité émotionnelle chez la caille : une approche intégrée du bien-être animal
}

\author{
S. RICHARD I, C. ARNOULD ${ }^{1}$, D. GUÉMENÉ2 ${ }^{2}$ C. LETERRIER ${ }^{1}$, S. MIGNON-GRASTEAU2, J.-M. FAURE \\ ${ }^{1}$ INRA, CNRS, Université de Tours, Haras Nationaux, UMR85 Physiologie de la Reproduction et des Comportements, \\ F-37380 Nouzilly, France \\ 2 INRA, UR83 Recherches Avicoles, F-37380 Nouzilly, France \\ Courriel : Sabine.Richard@tours.inra.fr
}

\begin{abstract}
Le bien-être des animaux d'élevage dépend notamment de leur capacité à ressentir des émotions et à les réguler. Il est donc essentiel de mieux connaître les mécanismes d'expression des émotions chez les animaux. Des travaux pluridisciplinaires (éthologie, physiologie, zootechnie) menés sur des modèles génétiques nous permettent de progresser dans la connaissance de la réactivité émotionnelle des oiseaux.

Le bien-être dépend de la façon dont l'animal perçoit son environnement, en particulier sur le plan émotionnel. En effet, le bien-être est une expérience

la caille : d'une part des lignées sélectionnées sur un comportement de peur, d'autre part des lignées sélectionnées sur la motivation sociale, ces

\section{1 / Lignées de cailles sélec- tionnées sur un comporte- ment de peur} subjective de l'animal, pour laquelle les émotions négatives sont limitées (peur, frustration...) et les émotions positives favorisées (confort, plaisir..., Veissier et al 2007). Ainsi l'étude de la réactivité émotionnelle, définie comme la prédisposition d'un individu à exprimer des réponses émotionnelles fortes, rapides et durables en réponse aux événements extérieurs (Boissy et al 2005), contribue à améliorer l'évaluation du bien-être en élevage (Boissy et al 2007). De plus, l'expression de certaines réactions émotionnelles, telles que les réactions de peur, peut s'avérer inadaptée en élevage et être source d'atteintes au bien-être des animaux. Il est donc important de comprendre les mécanismes qui conduisent à l'expression de ces réactions, afin d'aider à la conception de pratiques d'élevage qui limitent les perturbations émotionnelles ayant un impact négatif sur le bien-être.

L'une des voies d'approche envisagées pour limiter l'expression de réactions émotionnelles inadaptées en élevage est de sélectionner des animaux sur leur réactivité émotionnelle. Cette possibilité a été explorée à l'INRA de Nouzilly par Mills et Faure, qui ont initié à la fin des années 80 deux programmes de sélection génétique chez dernières permettant de prendre en compte l'influence de perturbations d'origine sociale sur la réactivité émotionnelle (Mills et Faure 1991). Cette dimension d'origine sociale est particulièrement importante chez les oiseaux d'élevage puisque la plupart sont élevés en grands groupes sociaux. La caille japonaise (Coturnix japonica) présente des caractéristiques comportementales et physiologiques proches de celles de la poule (Gallus gallus). Elle a été choisie comme modèle pour ces expériences de sélection génétique en raison de son faible intervalle entre générations. Ces lignées de cailles aux comportements extrêmes se sont avérées être de très bons modèles d'étude de la réactivité émotionnelle des oiseaux et elles ont été étudiées sous différents angles complémentaires : étude du comportement bien sûr, mais aussi des conséquences sur des paramètres de production, étude des corrélats hormonaux (activité de l'axe corticotrope), de l'activité du système nerveux autonome (variabilité de la fréquence cardiaque), du système nerveux central (recherche des régions du cerveau impliquées) et enfin, des bases génétiques sous-jacentes (recherche des régions du génome impliquées).

\section{1 / Les comportements de peur en élevage}

En conditions naturelles, les comportements de peur sont essentiels à la survie d'un individu car ils lui permettent d'échapper aux prédateurs et à de nombreux autres dangers. Cependant, l'expression de réactions de peur dans des environnements artificiels tels que les bâtiments d'élevage, où elles n'ont pas de caractère protecteur, peut se révéler préjudiciable pour l'individu et pour ses congénères. Par exemple, le déclenchement de réactions de panique conduit les poulets à courir dans n'importe quelle direction, à marcher sur leurs congénères et à s'entasser le long des murs, certains individus pouvant alors mourir par suffocation. De fortes réactions de peur face à l'homme peuvent aussi rendre les animaux difficiles à capturer. Il est donc souhaitable de réduire la peur chez les volailles en élevage. L'absence de peur est d'ailleurs l'une des cinq libertés fondamentales de l'animal d'élevage énoncées par le Farm Animal Welfare Council britannique depuis plusieurs décennies (Farm Animal Welfare Council 2007). Une sélection génétique d'animaux d'élevage aux comportements de peur 
réduits peut donc présenter un intérêt pratique puisqu'elle devrait permettre l'obtention d'animaux moins réactifs aux perturbations.

\section{2 / Le processus de sélection sur un comportement de peur : le comportement d'immobilité tonique}

Mills et Faure (1991) ont décrit en détail la procédure utilisée pour sélectionner des cailles sur la durée d'immobilité tonique. L'immobilité tonique est un état inné d'inhibition motrice passagère largement répandu dans le monde animal et qui peut être induit chez l'oiseau par une contention physique brève (Gallup 1974). Ce comportement est considéré comme le dernier recours dont les oiseaux disposent pour se défendre contre les prédateurs, en cas d'attaque par ces derniers. En effet, l'absence totale de mouvement de la part de la proie attrapée peut provoquer un désintérêt du prédateur, ce qui conférerait à l'immobilité tonique son caractère adaptatif.

Le comportement d'immobilité tonique est à la base d'un test classiquement utilisé pour évaluer la peur chez les oiseaux, car la durée de maintien de l'immobilité est considérée comme positivement corrélée à la peur de l'oiseau (Jones 1986). De plus, ce test présente l'avantage d'être non invasif, robuste, pratique et standardisable. Pour la sélection réalisée à l'INRA de Nouzilly, le comportement d'immobilité tonique est induit en maintenant la caille sur le dos pendant 10 secondes dans un support adapté (figure 1). Une fois relâchée, la caille peut rester immobile pendant un temps qui varie de quelques secondes à quelques heures. Le test est interrompu après 5 min d'immobilité si la caille ne s'est pas redressée.
Les cailles de la génération de départ de la sélection étaient issues du croisement réciproque de deux lignées commerciales. A chaque génération, une semaine après l'éclosion les individus sont observés dans le test d'immobilité tonique, mais aussi dans un test de motivation sociale (cf. $\S 2$ ). En effet, la sélection des reproducteurs tient compte à la fois de la durée d'immobilité tonique et de la motivation sociale des individus, de façon à conserver un niveau de motivation sociale moyen dans ces lignées. Deux lignées divergentes sont ainsi sélectionnées : une lignée de cailles à durée d'immobilité tonique longue $(\mathrm{E}+)$ et une lignée de cailles à durée d'immobilité tonique courte (E-). Une lignée témoin non sélectionnée est maintenue parallèlement. La divergence des deux populations est apparue dès la première génération et à partir de la 8 ème génération les écarts types des deux lignées ne se chevauchaient plus (figure 1). Bien que les tests de sélection soient réalisés sur des cailleteaux, les différences de durée d'immobilité tonique observées entre les lignées $\mathrm{E}$ - et $\mathrm{E}+$ se maintiennent à l'âge adulte (Launay et al 1993). De plus, quel que soit l'âge auquel le test est réalisé il n'apparaît pas de différence de durée d'immobilité tonique entre les sexes au sein de chaque lignée (Launay et al 1993).

\section{3 / Conséquences de la sélec- tion sur la durée d'immobilité tonique}

Nous présenterons dans cette section les résultats d'études dans lesquelles les cailles des lignées E- et E+ ont été comparées, avec l'objectif sous-jacent de mieux comprendre les manifestations de la peur chez les oiseaux, les mécanismes qui les régissent ainsi que leurs conséquences sur l'organisme (figure 2). La majorité de ces résultats a été présentée dans un article de synthèse (Faure et al 2006). Des citations spécifiques seront ajoutées pour les résultats n'ayant pas été rapportés par Faure et al (2006).

\section{a) Comportement}

Des mesures comportementales autres que le test d'immobilité tonique confirment que les cailles E- et E+ diffèrent par leur propension à exprimer des comportements de peur, ou émotivité. Ainsi, les cailles E+ s'immobilisent davantage (freezing) et sont moins actives que les cailles E- lorsqu'elles sont placées seules dans un environnement nouveau. De plus, les cailles E+ mettent plus de temps que les cailles Eà s'aventurer dans un espace inconnu, vide et fortement éclairé (Jones et al 1991). Lorsque les cailles sont placées dans une petite cage qui empêche tout déplacement, mais qui permet des mouvements limités de la tête et des pattes (cage de contention), les cailles E- se débattent davantage et font davantage de tentatives de fuite que les cailles $\mathrm{E}+$ (Faure et Mills 1998). Ces différents résultats montrent que face à une situation effrayante, les cailles de la lignée $\mathrm{E}+$ expriment davantage de comportements d'inhibition comportementale, tels que l'immobilité tonique ou le freezing. La réactivité à l'homme a été mesurée lors d'un ramassage manuel des cailles des deux lignées élevées ensemble au sol. Les cailles E- sont plus faciles à attraper que les cailles $\mathrm{E}+$ (Mills et Faure 2000), ce qui suggère que le comportement de fuite face à l'homme est plus marqué chez les cailles E+ que chez les cailles E-. En revanche, les comportements de peur des cailles des deux lignées sont similaires lorsqu'un stimulus inconnu (sonnerie ou objet) est présenté soudaine-

Figure 1. Sélection génétique sur un comportement de peur : photo d'un cailleteau pendant le test d'immobilité tonique et évolution de la durée d'immobilité tonique au cours des générations dans les différentes lignées.
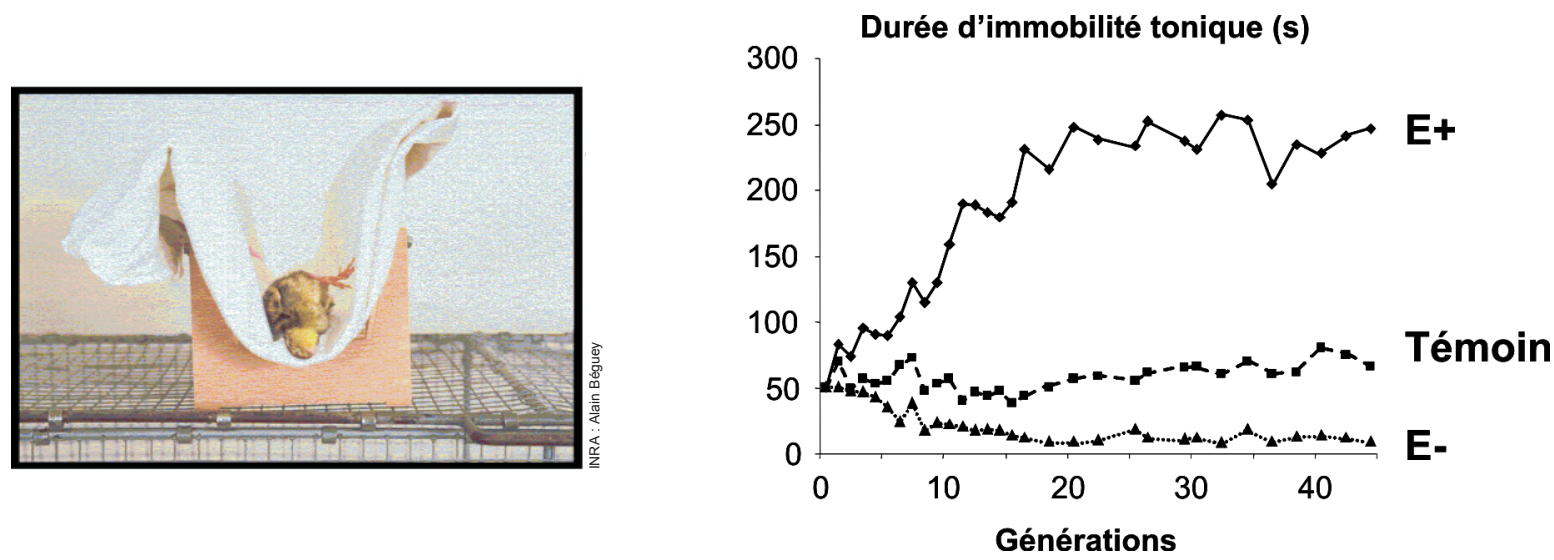
Figure 2. La recherche des mécanismes sous-tendant la réactivité émotionnelle des oiseaux vise à l'identification d'indicateurs de bien-être animal et de critères de sélection sur le comportement qui soient compatibles avec les impératifs de production.
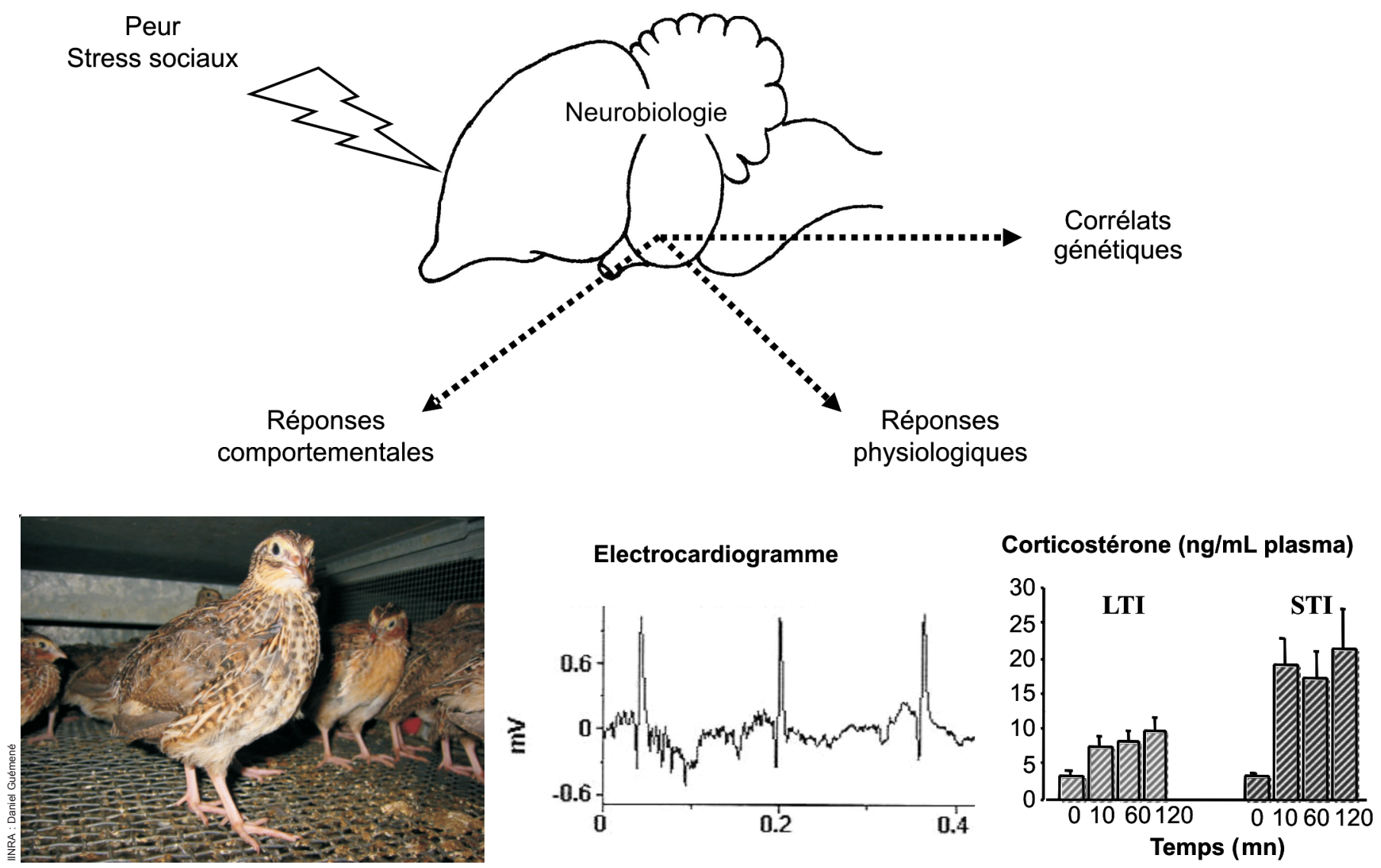

ment dans leur cage d'élevage avec une intervention humaine réduite (déclenchement du stimulus à distance : Richard et al 2007b, Valance et al 2007a). Des données récentes suggèrent en effet que les cailles E- et E+ se différencient essentiellement lorsqu'une une intervention humaine est impliquée dans le test, que les cailles soient placées en environnement nouveau ou non (Richard et al 2007a).

En termes de comportement reproducteur, les mâles E+ présentent un comportement sexuel plus actif que les mâles E-. Ce résultat est relativement surprenant dans la mesure où des niveaux élevés de peur sont généralement associés à une inhibition générale de nombreux autres comportements (Jones 1987). On aurait donc pu s'attendre à ce que les cailles $\mathrm{E}+$ aient un comportement sexuel moins actif que les cailles E-.

\section{b) Paramètres de production}

Les cailles E- sont plus lourdes, entrent en ponte plus jeunes et pondent plus d'œufs que les cailles E+. Toutefois, les œufs des cailles E- sont plus petits que ceux des cailles E+. Des études ont été réalisées pour comparer la qualité de la viande obtenue dans les deux lignées de cailles après trois types de stress mimant les conditions précédant classiquement l'abattage des volailles : contention, jeûne et transport. Dans les trois conditions, le $\mathrm{pH}$ du muscle pectoral $24 \mathrm{~h}$ post mortem est significativement plus élevé chez les cailles E+ que chez les cailles E-. De plus, après le stress de contention, le muscle pectoral des cailles $\mathrm{E}+$ montre une plus forte perte en eau que celui des cailles E-. Ces deux paramètres suggèrent que suite à un stress la qualité de la viande des cailles $\mathrm{E}+$ est moins bonne que celle des cailles E-. Globalement, ces résultats semblent indiquer que la sélection d'oiseaux moins émotifs est compatible avec le maintien de bonnes performances de production.

\section{c) Axe corticotrope}

L'activation de l'axe corticotrope (hypothalamus-hypophyse-surrénales) est l'un des principaux mécanismes physiologiques permettant de réguler l'homéostasie de l'organisme en réponse à un événement stressant. Son activation se traduit chez les oiseaux par la libération de corticostérone dans le sang et le dosage de cette hormone permet d'évaluer l'activité de l'axe corticotrope d'un individu dans une condition donnée. Sans stimulation particulière, le taux de corticostérone plasmatique ne diffère pas entre les deux lignées.

Un stress modéré, tel que l'introduction d'un objet nouveau dans la cage des cailles ou l'isolement social dans un environnement inconnu, entraîne une légère augmentation de la corticostéronémie, similaire chez les deux lignées de cailles. En revanche lors d'un stress plus intense, tel qu'une contention manuelle ou en cage (description $\S$ 1.3.a), l'augmentation de la corticostéronémie est significativement plus marquée chez les cailles E- que chez les cailles E+. De même, à la suite d'une heure de transport, seules les cailles E- présentent une augmentation du niveau de corticostérone plasmatique, la corticostéronémie des cailles E+ ne s'élevant pas au-dessus du niveau de base. Ces différences peuvent être mises en relation avec les réponses comportementales des cailles des deux lignées. En effet, la plus grande activité des cailles E- en cage de contention (cf. $\S$ 1.3.a) pourrait expli- 
quer l'augmentation supérieure du taux de corticostérone plasmatique observée chez ces cailles. Cependant, l'activité comportementale à elle seule ne peut pas expliquer l'intégralité des différences observées au niveau endocrinien puisque si la contention est longue $(2 \mathrm{~h})$, les cailles E- se débattent de moins en moins au cours du temps, alors que leur taux de corticostérone plasmatique reste élevé jusqu'à la fin de la contention. Les relations entre comportement et corticostéronémie ont été spécifiquement évaluées dans une étude menée sur un croisement de deuxième génération entre les cailles $\mathrm{E}-$ et $\mathrm{E}+$. Cette étude a montré l'indépendance entre le taux de corticostérone plasmatique après contention et différentes variables comportementales, dont la durée d'immobilité tonique (MignonGrasteau et al 2003). Ces résultats illustrent la complexité des relations entre les réponses comportementales et les réponses physiologiques d'un individu et la nécessité de poursuivre les investigations afin de mieux comprendre les mécanismes mis en jeu.

Des expériences pharmacologiques ont été menées dans le but d'identifier à quel(s) niveau(x) de l'axe corticotrope se situaient les différences de réactivité observées entre les deux lignées : hypothalamus, hypophyse ou surrénales. La réactivité des glandes surrénales, testée par injection d'hormone adénocorticotrope (ACTH), ne diffère pas entre les lignées E- et E+. En revanche, la stimulation de l'hypophyse par injection de corticolibérine (CRF) induit une libération de corticostérone plasmatique supérieure chez les cailles E(Hazard et al 2007). Par conséquent, il est possible qu'un effet différentiel du CRF au niveau hypophysaire puisse expliquer les différences de réactivité de l'axe corticotrope observées. Ces différences de réactivité pourraient également résulter de différences entre les lignées en termes de perception des situations au niveau central.

\section{d) Système nerveux autonome}

Le système nerveux autonome, composé du système nerveux sympathique et du système nerveux parasympathique, est également très sollicité par les situations génératrices d'émotions. Il prépare l'animal à l'action et pour cela, régule l'activité de nombreux organes, dont le cœur qui est accéléré par le système orthosympathique, en particulier en cas de stress, et ralenti par le système parasympathique. L'étude de l'activité cardiaque des cailles a permis de mettre en évidence des différences de fonctionnement du système nerveux autonome entre les deux lignées. Pour ces travaux, les cailles sont équipées d'implants de télémétrie, qui permettent d'enregistrer les électrocardiogrammes à distance, sans perturber l'animal. La fréquence cardiaque est soumise à des variations extrêmement rapides et une analyse détaillée de cette variabilité permet d'évaluer les influences respectives des branches sympathique et parasympathique du système nerveux autonome, ces influences déterminant l'équilibre sympatho-vagal.

Lorsque les cailles ne sont soumises à aucune stimulation particulière, dans la plupart des expériences la fréquence cardiaque de base est similaire chez les cailles E+ et E-, ainsi que chez les cailles de la lignée témoin non sélectionnée. Cependant, une étude de blocage pharmacologique a révélé que le cœur des cailles E- et des cailles de la lignée témoin est sous influence prédominante du système parasympathique, alors que chez les cailles E+ les influences sympathiques et parasympathiques sur le cour s'équilibrent. Cela pourrait expliquer que dans certaines études la fréquence cardiaque basale soit plus faible chez les cailles E- que chez les cailles E+ (Valance et al 2007b).

Le système nerveux autonome des cailles a également été étudié en situation génératrice de peur. Ainsi pendant l'induction du comportement d'immobilité tonique, on observe une forte augmentation de l'activité sympathique et une diminution de l'activité parasympathique dans les trois lignées (E-, E+ et témoin), mais ces changements sont plus amples chez les cailles E- (Valance et al 2007c). L'existence d'une relation entre durée d'immobilité tonique et réponse du système nerveux autonome en situation de stress est suggérée par l'observation de différences entre les lignées E- et E+ lors de l'audition d'un son inhabituel : dans cette situation, les cailles des deux lignées montrent une forte activation sympathique, mais chez les cailles E- le système parasympathique est également activé (Valance et al 2007a). Dans cette lignée, l'activation supplémentaire du système parasympathique pourrait être liée à la prédominance de ce système au repos chez ces cailles.

La sélection sur la durée d'immobilité tonique s'est donc accompagnée de changements dans le contrôle de l'activité cardiaque par le système nerveux autonome, à la fois en situation basale et en réponse à une stimulation.

\section{e) Système nerveux central}

La comparaison des cailles E+ et Esur le plan neurobiologique a été essentiellement focalisée sur une région du cerveau d'oiseau connue pour son implication dans le contrôle des comportements de peur et autrefois appelée archistriatum. Cette région englobe deux structures, l'arcopallium et l'amygdale palliale postérieure, mais elle a été étudiée dans sa globalité car la limite entre les deux subdivisions n'est pas bien établie. La lésion de cette région n'entraîne pas de modification notable du comportement des cailleteaux E-, mais réduit significativement les réponses comportementales de peur des cailleteaux E+. La lésion de cette région chez le jeune modifie également le comportement des cailles E+ à l'âge adulte : les cailles ayant subi la lésion sont plus faciles à capturer et présentent moins d'évitement de personnes, d'objets et d'aliments nouveaux. De plus, la région englobant l'arcopallium et l'amygdale palliale postérieure est significativement plus volumineuse chez les cailles E- que chez les cailles $\mathrm{E}+$. Ce résultat pourrait suggérer le développement préférentiel d'un groupe de neurones qui inhibent les comportements de peur dans l'arcopallium ou l'amygdale palliale postérieure des cailles E-. Des résultats morphologiques récents (données non publiées) suggèrent qu'une telle région inhibitrice pourrait se trouver dans la partie caudale de l'arcopallium/amygdale palliale postérieure. Cette hypothèse est renforcée par les résultats d'une autre étude menée chez les cailles $\mathrm{E}+$, indiquant que des lésions restreintes à la partie caudale de l'arcopallium/amygdale palliale postérieure induisent une augmentation de l'expression de certains comportements de peur (SaintDizier et al 2007).

Une partie des différences comportementales et physiologiques observées entre les lignées E+ et E- résulte vraisemblablement de différences au niveau du cerveau, dans la mesure où ce dernier coordonne les réactions périphériques. Bien que les relations causales entre les différents paramètres ne soient pas encore établies, les lignées $\mathrm{E}+$ et E- constituent un excellent modèle pour l'investigation des circuits de contrôle des comportements de peur dans le cerveau d'oiseau. En effet, la comparaison d'animaux présentant des comportements extrêmes facilite la 
mise en évidence des mécanismes neurobiologiques sous-jacents.

\section{4 / Mécanismes génétiques impliqués}

Les estimations de l'héritabilité de la durée d'immobilité tonique, réalisées sur les 8 premières générations, varient entre 0,2 et 0,3 . Une recherche de régions du génome (QTL = Quantitative Trait Loci) associées aux comportements de peur a été réalisée sur un croisement de deuxième génération entre les cailles E- et E+. Cinq QTL significatifs au niveau du génome ont été identifiés, dont un lié à la durée d'immobilité tonique. Il est intéressant de noter que ce dernier est très proche d'un QTL moins significatif associé au comportement en open-field, ces deux QTL pouvant être identiques. Ce résultat suggère que des gènes modulant la propension à exprimer des comportements de peur pourraient se trouver dans cette région du génome. En revanche, aucun QTL lié à la fois à la durée d'immobilité tonique et à la réponse en corticostérone plasmatique après contention n'a pu être identifié (Beaumont et al 2005). Des études ultérieures seront nécessaires pour affiner la recherche des gènes impliqués dans le contrôle des comportements de peur chez les oiseaux.

\section{2 / Lignées de cailles sélec- tionnées sur la motivation sociale}

\section{1 / Les comportements sociaux en élevage}

Les volailles de chair (poulets, pintades, dindes, canards,...) et les poules pondeuses en volière et au sol sont élevées en groupes de plusieurs milliers, voire plusieurs dizaines de milliers d'individus. Or le comportement de ces espèces d'élevage, dont les ancêtres vivent en petits groupes sociaux, n'est pas toujours adapté à ce mode de production. Le grand nombre d'individus est de plus généralement associé à une forte densité ; ces conditions perturbent l'établissement de liens sociaux stables, peuvent limiter l'accès aux ressources (eau, aliment, chauffage) et supposent une grande tolérance entre les individus. Le problème de la densité est d'autant plus marqué qu'en élevage les individus se répartissent de façon hétérogène dans le bâtiment, ce qui induit des densités locales plus élevées que la densité moyenne (Fraysse et al
2001). Différents comportements sociaux indésirables sont observés en élevage, tels que l'agressivité, le picage de plumes ou le cannibalisme, à l'origine de blessures et de la mort de certains individus (Jones et Mills 1999). Une sélection génétique sur la motivation sociale a donc été entreprise avec l'espoir d'obtenir des animaux plus tolérants aux fortes densités et aux grands groupes.

\section{2 / Le processus de sélection sur la motivation au regroupe- ment social}

La procédure utilisée pour sélectionner des cailles sur la motivation au regroupement social a été décrite en détail par Mills et Faure (1991). La motivation sociale est estimée par la distance parcourue par un cailleteau sur un tapis roulant pour rejoindre un groupe de congénères (figure 3 ).

La population de départ utilisée pour cette sélection était la même que celle utilisée pour la sélection sur la durée d'immobilité tonique. A chaque génération, les cailleteaux sont testés à l'âge d'une semaine, à la fois dans le test du tapis roulant et dans le test d'immobilité tonique (cf. $\S 1$ ). Ainsi, la sélection des reproducteurs est réalisée sur la base d'un index composite qui tient compte non seulement de la motivation sociale, mais aussi de la durée d'immo- bilité tonique des individus, de façon à conserver un niveau d'émotivité moyen dans ces lignées. Deux lignées divergentes sont ainsi sélectionnées : une lignée de cailles à forte motivation au regroupement social $(\mathrm{S}+)$ et une lignée de cailles à faible motivation au regroupement social (S-). Une lignée témoin non sélectionnée est maintenue parallèlement. La divergence des deux populations est apparue dès la première génération et s'est accentuée au cours des générations suivantes (figure 3). A

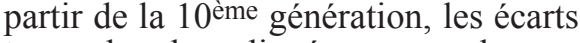
types des deux lignées ne se chevauchaient plus. Les différences de motivation au regroupement social entre les lignées $\mathrm{S}$ - et $\mathrm{S}+$ se maintiennent à l'âge adulte (Launay et al 1993).

\section{3 / Conséquences de la sélec- tion sur la motivation au regrou- pement social}

La majorité des résultats concernant les lignées $\mathrm{S}+$ et $\mathrm{S}$ - a été présentée dans un article de synthèse (Jones et Mills 1999). Des citations spécifiques seront ajoutées pour les résultats qui n'ont pas été présentés dans cet article. L'observation comportementale des cailles $\mathrm{S}+$ et $\mathrm{S}-$ a permis de confirmer que les comportements des cailles de ces lignées diffèrent dès lors que la situation comprend un aspect social. Ainsi, les cailles $\mathrm{S}+$ sont plus sensibles à l'isolement social et les distances

Figure 3. Sélection génétique sur la motivation au regroupement social : photo d'un cailleteau pendant le test du tapis roulant et évolution de la distance parcourue pour rejoindre des congénères dans ce test au cours des générations dans les différentes lignées.
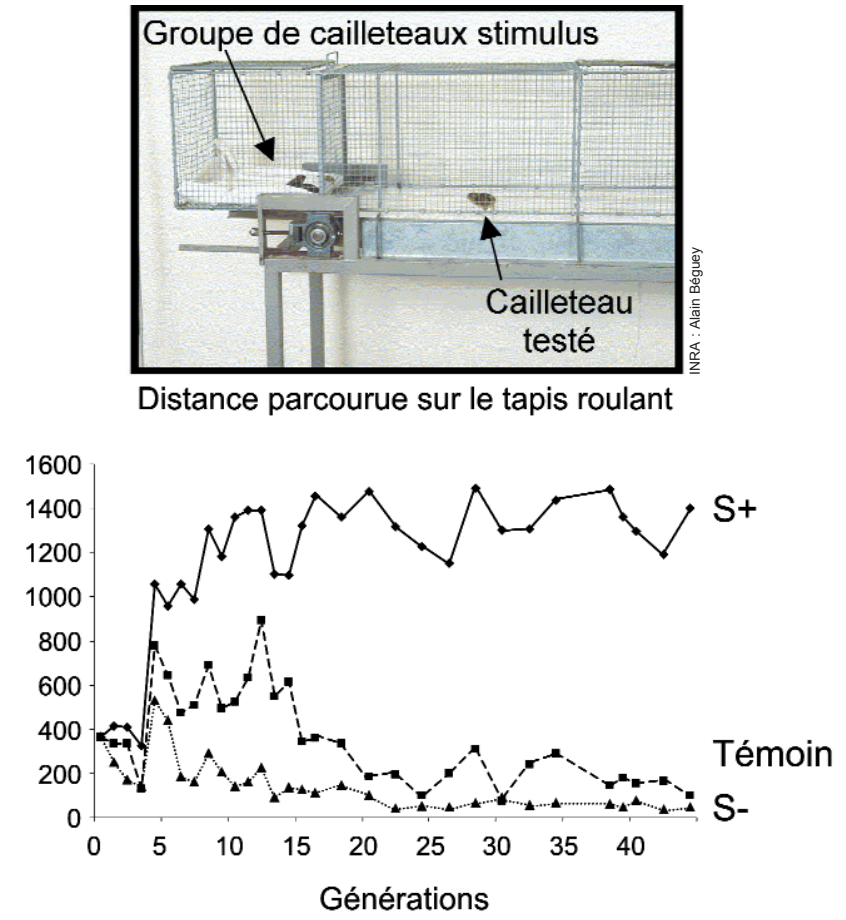
interindividuelles à l'âge d'une et 3 semaines sont plus faibles dans un groupe de cailles $\mathrm{S}+$ que dans un groupe de cailles S-. Lorsqu'elles sont élevées dans un dispositif où elles ont accès soit à un espace recouvert de litière et contenant eau et aliment mais ne permettant pas de voir de congénères, soit à un espace vide, au sol grillagé, mais qui permet d'interagir avec un congénère au travers d'un grillage, les cailles $\mathrm{S}+$ âgées de 4 et 6 semaines passent plus de temps que les cailles Sdans ce deuxième compartiment. Des travaux récents indiquent que lorsqu'ils sont placés dans une situation de choix entre un individu inconnu et un indivi$\mathrm{du}$ familier, les cailleteaux $\mathrm{S}+$ âgés d'une semaine rechercheraient le contact social quel que soit le partenaire, alors que les cailleteaux S- seraient plus sensibles à la qualité du partenaire (Schweitzer et al 2007). Enfin, les cailles $\mathrm{S}+$ sont également plus sensibles à la facilitation sociale : elles ingèrent plus rapidement un aliment nouveau si elles voient une autre caille ingérer cet aliment. A l'âge adulte, les cailles mâles $\mathrm{S}+$ présentent également un comportement sexuel plus actif que les cailles mâles S-.

Cependant, les cailles à forte motivation sociale ne sont pas nécessairement bien adaptées aux conditions d'élevage. En effet, à l'âge adulte elles présentent plus de comportements agressifs que les cailles S- envers une caille non familière (Arnould et al 2002). Les cailles S-, qui ont une motivation sociale faible et qui semblent de façon générale plus indifférentes envers leurs congénères (François et al 2000), pourraient donc finalement s'avérer mieux adaptées aux élevages en grand groupe et à forte densité.

La réactivité des cailles $\mathrm{S}+$ et $\mathrm{S}$ - face à l'homme a été testée dans le même test de ramassage manuel que celui uti- lisé pour l'étude des lignées E- et E+ (cf. § 1.3.a) : les cailles de la lignée S+ sont plus faciles à attraper que les cailles S- (Mills et Faure 2000), ce qui suggère que la sélection pour la motivation sociale peut avoir une influence sur les relations homme-animal. Une telle relation peut avoir des conséquences pratiques en élevage et serait donc importante à prendre en compte si une sélection sur la motivation sociale était envisagée commercialement.

\section{Conclusion}

Les sélections génétiques sur la peur et sur la motivation sociale ont initialement été entreprises par Mills et Faure pour étudier la faisabilité de telles sélections et les conséquences qu'elles pouvaient avoir, non seulement pour le bien-être animal mais aussi en termes de production. La faisabilité est démontrée dans les deux cas et les conséquences d'une sélection d'oiseaux aux comportements de peur réduits semblent compatibles avec une prise en compte de ce critère dans les schémas de sélection commerciale. En ce qui concerne les comportements sociaux, contrairement à l'hypothèse initiale, les cailles à forte motivation sociale semblent moins bien adaptées aux conditions d'élevage que les cailles à faible motivation sociale, plus tolérantes vis-à-vis de leurs congénères. Des travaux complémentaires sont nécessaires pour évaluer si la sélection d'oiseaux à faible motivation sociale est envisageable en termes de production. L'introduction de critères comportementaux dans les schémas de sélection sera facilitée par la mise au point de méthodes rapides d'évaluation de ces critères sur un grand nombre d'individus. A terme, les différents travaux menés sur les lignées de cailles présentées dans cet article pourraient conduire à l'identification de marqueurs - génétiques, par exem- ple - qui simplifieraient la prise en compte des caractères comportementaux dans les schémas de sélection.

Outre leur intérêt potentiel en termes de génétique avicole, les lignées de cailles sélectionnées sur la peur et sur la motivation sociale se sont révélées comme de puissants modèles d'étude des mécanismes sous-tendant ces comportements. En effet, la comparaison d'animaux aux phénotypes extrêmes fa-cilite la mise en évidence des mécanismes sous-jacents. Les travaux menés sur les cailles des lignées E- et E+ ont d'ores et déjà permis d'identifier des corrélats physiologiques, neurobiologiques et génétiques associés à l'expression de comportements de peur chez les oiseaux. Parallèlement, l'étude comportementale des cailles $\mathrm{S}+$ et $\mathrm{S}$ montre que ces lignées constituent un modèle de choix pour étudier les déterminants des comportements sociaux chez les oiseaux. La poursuite des travaux pluridisciplinaires engagés sur ces quatre lignées vise notamment à identifier des indicateurs de bien-être. La prise en compte de plusieurs paramètres de la réactivité émotionnelle des oiseaux permet en effet de mieux appréhender les émotions qu'ils peuvent ressentir dans différentes situations. Les indicateurs mis en évidence pourront servir à comparer le bien-être des oiseaux dans différents types d'élevages.

\section{Remerciements}

M. Couty et J.-M. Hervouët sont remerciés pour leur implication technique dans l'entretien et la sélection des lignées de cailles présentées dans cet article.

\section{Références}

Arnould C., Quekenborn D., Mills A.D., Faure J.M., 2002. Behaviour and emotional reactivity of quails reared in stable or unstable groups. $36^{\text {th }}$ Int. Cong. ISAE, 6-10 August, Wageningen, The Netherlands, 170.

Beaumont C., Roussot O., Feve K., Vignoles F., Leroux S., Pitel F., Faure J.M., Mills A.D., Guémené D., Sellier N., Mignon-Grasteau S., Le Roy P., Vignal A., 2005. A genome scan with AFLPTM markers to detect fearfulness-related QTLs in Japanese quail. Anim. Genet., 36, 401407.
Boissy A., Bouix J., Orgeur P., Poindron P., Bibé B., Le Neindre P., 2005. Genetic analysis of emotional reactivity in sheep: effects of the genotypes of the lambs and of their dams. Genet. Sel. Evol., 37, 381-401.

Boissy A., Arnould C., Chaillou E., Colson V., Désiré L., Duvaux-Ponter C., Greiveldinger L., Leterrier C., Richard S., Roussel S., Saint-Dizier H., Meunier-Salaün M.C., Valance D., 2007. Emotions et cognition : stratégie pour répondre à la question de la sensibilité des animaux. INRA Prod. Anim., 20, 17-21.
Farm Animal Welfare Council, 2007. Annual review 2006/2007. Farm Animal Welfare Council, London, UK, 16 p.

Faure J.M., Mills A.D., 1998. Improving the adaptability of animals by selection. In: Genetics and the behavior of domestic animals, Grandin T. (Ed). Academic Press, San Diego, USA, 235264.

Faure J.M., Arnould C., Beaumont C., Guémené D., Leterrier C., Mills A.D., Richard S., 2006. Consequences of selection for fear in Japanese quail. Arch. Geflügelkd., 70, 216-222. 
Francois N., Decros S., Picard M., Faure J.M., Mills A.D., 2000. Effect of group disruption on social behaviour in lines of Japanese quail (Coturnix japonica) selected for high or low levels of social reinstatement behaviour. Behav. Process., 48, 171-181.

Fraysse V., Mirabito L., Arnould C., 2001. Utilisation de l'espace par des poulets de chair «standards» et activité au niveau des abreuvoirs et des mangeoires selon leur localisation. 4èmes Journ. Rech. Avicole, 27-29 mars, Nantes, France, 105-108.

Gallup G.G., 1974. Animal hypnosis: factual status of a fictional concept. Psychol. Bull., 81, 836-853.

Hazard D., Couty M., Guémené D., 2007. Characterization of CRF, AVT, and ACTH cDNA and pituitary-adrenal axis function in Japanese quail divergently selected for tonic immobility. Am. J. Physiol. Regul. Integr. Comp. Physiol., 293, R1421-1429.

Jones R.B., 1986. The tonic immobility reaction of the domestic fowl: a review. Wld Poultry Sci. J., 42, 82-97.

Jones R.B., 1987. The assessment of fear in the domestic fowl. In: Cognitive aspects of social behaviour in the domestic fowl. Zayan R., Duncan I. J. H. (Eds). Elsevier, Amsterdam, 40-81.

Jones R.B., Mills A.D., 1999. Divergent selection for social reinstatement behaviour in Japanese quail: effects on sociality and social discrimination. Poultry Avian Biol. Rev., 10, 213-223.

Jones R.B., Mills A.D., Faure J.M., 1991. Genetic and experiential manipulation of fear- related behavior in Japanese quail chicks (Coturnix coturnix japonica). J. Comp. Psychol., 105, 15-24.

Launay F., Mills A.D., Faure J.M., 1993. Effects of test age, line and sex on tonic immobility responses and social reinstatement behaviour in Japanese quail Coturnix japonica. Behav. Process., 29, 1-16.

Mignon-Grasteau S., Roussot O., Delaby C., Faure J.M., Mills A., Leterrier C., Guémené D., Constantin P., Mills M., Lepape G., Beaumont C., 2003. Factorial correspondence analysis of fear-related behaviour traits in Japanese quail. Behav. Process., 61, 69-75.

Mills A.D., Faure J.M., 1991. Divergent selection for duration of tonic immobility and social reinstatement behavior in Japanese quail (Coturnix coturnix japonica) chicks. J. Comp. Psychol., 105, 25-38.

Mills A.D., Faure J.M., 2000. Ease of capture in lines of Japanese quail (Coturnix japonica) subjected to contrasting selection for fear or sociability. Appl. Anim. Behav. Sci., 69, 125134.

Richard S., Land N., Leterrier C., Saint-Dizier H., 2007a. Une sélection génétique sur un comportement de peur n'affecte pas tous les comportements de peur. 2 èmes Journ. Animation Scienti. Départ. PHASE, INRA, 22-24 octobre 2007, Tours, France, 34.

Richard S., Wacrenier-Ceré N., Hazard D., Saint-Dizier H., Arnould C., Faure J.M., 2007b. Behavioural and endocrine fear responses in Japanese quail upon presentation of a novel object in the home cage. Behav. Process. doi:10.1016/j.beproc.2007.07.005.
Saint-Dizier H., Leterrier C., Lévy F., Richard S., 2007. Subdivisions of the arcopallium are differentially involved in the control of fear behaviour in the Japanese quail. $39^{\text {th }}$ Ann. Gen. Meet. Eur. Brain Behav. Soc., September 15-19, Trieste, Italy. Neural Plasticity, 72-73.

Schweitzer C., Poindron P., Arnould C., 2007. Discrimination entre un individu familier et un inconnu chez des cailles japonaises (Coturnix japonica) à forte ou faible motivation sociale âgées d'une semaine ou de six semaines. $41^{\text {ème }}$ Coll. Soc. Fr. Etude du Comportement Animal, 18-20 avril, Université Paris 13, Villetaneuse, France, 17.

Valance D., Boissy A., Després G., Constantin P., Leterrier C., 2007a. Emotional reactivity modulates autonomic responses to an acoustic challenge in quail. Physiol. Behav., 90, 165-171.

Valance D., Després G., Boissy A., MignonGrasteau S., Constantin P., Leterrier C., 2007b. Genetic selection on a behavioural fear trait is associated with changes in heart rate variability in quail. Genes Brain Behav., 6, 339-346.

Valance D., Després G., Richard S., MignonGrasteau S., Leman S., Boissy A., Faure J.M., Leterrier C., 2007c. Changes in heart rate variability during a tonic immobility test in quail. Physiol. Behav., doi: 10.1016/j.physbeh. 2007.10.011.

Veissier I., Beaumont C., Lévy F., 2007. Les recherches sur le bien-être animal : buts, méthodologie et finalité. INRA Prod. Anim., 20, 3-10.

\section{Résumé}

Certaines réactions émotionnelles, telles que les réactions de peur, peuvent être inadaptées en élevage, sources d'atteintes au bien-être des animaux. Chez la caille, l'obtention de lignées génétiques divergentes sélectionnées d'une part sur un comportement de peur, d'autre part sur la motivation sociale, a démontré la faisabilité de sélections sur des composantes de la réactivité émotionnelle. Les cailles sélectionnées sur un comportement de peur diffèrent non seulement par leur propension générale à exprimer des comportements de peur, mais aussi en termes de qualité de la viande, de réactivité de l'axe corticotrope, d'activité du système nerveux autonome et au niveau neurobiologique. Une région du génome a été identifiée comme potentiellement impliquée dans les différences observées entre les lignées. Par ailleurs, les cailles sélectionnées pour leur faible motivation sociale se sont montrées plus indifférentes à leur environnement social que les cailles sélectionnées pour leur forte motivation sociale. Ces lignées de cailles se sont révélées comme de puissants modèles d'étude des mécanismes sous-tendant la réactivité émotionnelle chez les oiseaux. Les travaux pluridisciplinaires engagés sur ces cailles ont pour buts d'évaluer les conséquences de ces sélections, non seulement pour le bien-être animal mais aussi en termes de production, et d'identifier des indicateurs de bien-être qui pourront servir à comparer différents systèmes d'élevage des volailles.

\section{Abstract}

\section{Emotional reactivity in the quail: an integrated approach to animal welfare}

In farm animals, emotional reactions such as fear behaviour may be inadequate and may compromise animal welfare. Two programmes of divergent genetic selection in the Japanese quail have demonstrated the feasibility of selection on components of emotional reactivity: fear behaviour on the one hand and social reinstatement behaviour on the other hand. Divergent selection of the quail on a fear behaviour trait has been accompanied by differences in general fearfulness, meat quality, hypothalamo-pituitary-adrenal axis reactivity, autonomic nervous system activity and central nervous system characteristics. A region of the genome has been identified as being potentially involved in the observed differences. The second selection programme has shown that the quail selected for low social reinstatement behaviour is more indifferent to their social environment than the quail selected for high social reinstatement behaviour. These quail lines constitute a powerful tool to study the mechanisms of emotional reactivity in birds. The aims of the multidisciplinary work being currently carried out on these quail are to assess the consequences of these selection programmes, not only in terms of animal welfare but also in terms of animal production, and to identify welfare indicators that may be used to compare different farming practices in poultry production.

RICHARD S., ARNOULD C., GUÉMENÉ D., LETERRIER C., MIGNON-GRASTEAU S., FAURE J.-M., 2008. Etude de la réactivité émotionnelle chez la caille : une approche intégrée du bien-être animal. INRA Prod. Anim., 21, 71-78. 
